\title{
The Prolongation Structures for the System of the Reaction-Diffusion Type
}

\author{
Xiaojuan Duan \\ Faculty of Applied Mathematics, Xiamen University of Technology, Xiamen, China \\ Email: 2010111012@xmut.edu.cn
}

How to cite this paper: Duan, X.J. (2017) The Prolongation Structures for the System of the Reaction-Diffusion Type. Journal of Applied Mathematics and Physics, 5, 92100.

http://dx.doi.org/10.4236/jamp.2017.51010

Received: January 3, 2017

Accepted: January 21, 2017

Published: January 24, 2017

Copyright $\odot 2017$ by author and Scientific Research Publishing Inc. This work is licensed under the Creative Commons Attribution International License (CC BY 4.0).

http://creativecommons.org/licenses/by/4.0/

\begin{abstract}
Equations of the reaction-diffusion type are very well known and have been extensively studied in many research areas. In this paper, the prolongation structures for the system of the reaction-diffusion type are investigated from theory of coverings. The realizations and the classifications of the one-dimensional coverings of the system are researched. And the corresponding conservation law of the one-dimensional Abelian coverings is concluded, which is closely connected with the symmetry of the system by Noether theorem.
\end{abstract}

\section{Keywords}

Prolongation Structure, Reaction-Diffusion, Realization, Abelian Covering

\section{Introduction}

Equations of the reaction-diffusion type have been widely studied in many research areas [1] [2]. One of the reaction-diffusion types has received considerable attention,

$$
\left\{\begin{array}{l}
u_{t}-u_{x x}+2 u^{2} v-2 k u=0 \\
v_{t}+v_{x x}-2 v^{2} u+2 k v=0
\end{array}\right.
$$

which has been applied in biological systems, chemical autocatalysis, and also in the gauge formulation of the $(1+1)$-dimensional gravity [3]. Moreover, the geometrical equivalent counterpart of the system (1) is the modified Heisenberg Ferromagnetic (HF) equation [4] [5].

About the prolongation structure of the system (1), some results have been obtained. Alfinito et al. [1] used Wahlquist-Estabrook (WE) prolongation structure theory proposed by Wahlquist and Estabrook [6] [7] and carried out the detailed integrable analysis. They showed that (1) allowed an incomplete prolongation algebra admitting an infinite-dimensional realization of the Kac- 
Moody type. Later following my advisor Zhao W Z's suggestion, we investigated the inhomogeneous extension of the system (1) by the covariant prolongation structures theory [2]. We constructed $\operatorname{sl}(2, R) \times R(\rho(t))$ prolongation structure and gave the corresponding Ablowitz-Kaup-Newell-Segur (AKNS-) type equations and Bäcklund transformation. In 2012 Krasil'shchik and Verbovetsky [8] gave an overview of the recent results on the geometry of partial differential equations (PDEs) in application to integrable systems. These results are essentially based on the geometrical approach to partial differential equations developed since 1970s by A.M. Vinogradov and his school [9] [10] [11] [12] [13]. The approach is called the theory of coverings, which treats a PDE as an (infinite-dimensional) submanifold in the space $J^{\infty}(\pi)$ of infinite jets for a bundle $\pi: E \rightarrow M$ whose sections play the role of unknown functions (fields). This attitude allows applying to PDEs powerful techniques of differential geometry and homological algebra. Readers can refer [8] for more information. It is noticed that the WE prolongation structures are an essentially special type of coverings [13] [15]. Cheng and He successfully gave the realizations and classifications of one-dimensional coverings of the MB (modified Boussinesq) system by using the theory. Moreover, they also gave the sufficient and necessary conditions for a vector to be a nonlocal symmetry of the MB system. Hence we want to apply the theory of coverings to the system (1) to give some new integrable information.

The paper is organized as follows. In Section 2, we review some basic notations and theorems due to [13] [14] [15]. In Section 3, we apply the theory to the system (1) and obtain the realization and classifications of one-dimensional coverings of the system. Also we give the corresponding conservation law for onedimensional Abelian coverings. In Section 4, we give a conclusion.

\section{Basic Definitions and Statements}

In this section, we mainly recall some definitions and theorems in [14] [15]. For an equation $\varepsilon$ in $n$ independent variables $x_{i}$ and $m$ unknown dependent functions $u^{j}$, we consider the jet space $J^{\infty}(n, m)$ with the coordinates $x_{i}, u_{\sigma}^{j}$, where $\left(x_{1}, \cdots, x_{n}, j=1, \cdots, m\right)$, and $\sigma=\left(i_{1}, i_{2}, \cdots,\left|i_{\sigma}\right|\right), 0 \leq i_{k} \leq n$ is a multi-index of finite, but unlimited length $\left|i_{\sigma}\right|$. Denote by $\pi: J^{\infty}(n, m) \rightarrow \mathbb{R}^{n}$ the projection to the space of space of independent variables $\left(x_{1}, \cdots, x_{n}\right)$.

The vector fields

$$
D_{i}=\frac{\partial}{\partial x_{i}}+\sum_{j, \sigma} u_{\sigma+1}^{j} \frac{\partial}{\partial u_{\sigma}^{j}}
$$

are called total derivatives. They commute each other, i.e., $\left[D_{i}, D_{j}\right]=0$ on $J^{\infty}(m, n)$ everywhere. $D_{i}, i=1, \cdots, n$ span a distribution on $J^{\infty}(m, n)$ which is called Cartan distribution and is denoted by $\mathcal{C}$.

Let a system of PDEs be given by

$$
F_{\alpha}\left(x_{i}, \cdots, u_{\sigma}^{j}, \cdots,\right)=0, \quad 1 \leq \alpha \leq s, \quad 1 \leq i \leq n, 1 \leq j \leq m .
$$

Then we consider all its differential consequences, or prolongation of (3)

$$
D_{\sigma} F_{\alpha}=0, \quad 1 \leq \alpha \leq s, \quad|\sigma| \geq 0
$$


where $D_{\sigma}=D_{\left(i_{1}, i_{2}, \cdots, i_{k}\right)}$ for $\sigma=\left(i_{1}, i_{2}, \cdots, i_{k}\right)$. The hyper surface defined by (4) is denoted by $\varepsilon \subset J^{\infty}(n, m)$. The vector fields $D_{i}$ are tangent to $\varepsilon$, and their restriction to $\varepsilon$ will be denoted by the same symbol $D_{i}$. They span a distribution on $\varepsilon$ which is called Cartan distribution of $\varepsilon$ and is denoted by $\mathcal{C}_{\varepsilon}$.

Consider another submanifold $\tilde{\varepsilon} \subset J^{\infty}(n, m)$ with the same independent variables $\left(x_{1}, \cdots, x_{n}\right)$. And $\tilde{\varepsilon}$ has an integrable distribution $\tilde{\mathcal{C}}$, i.e., $[\tilde{\mathcal{C}}, \tilde{\mathcal{C}}]=0$. A smooth surjection $\tau: \tilde{\varepsilon} \rightarrow \varepsilon$ is called a differential covering (or simply a covering) of $\varepsilon$ by $\tilde{\varepsilon}$ if its differential takes the Cartan distribution on $\tilde{\varepsilon}$ to that on $\tilde{\varepsilon}$, i.e., $\tau_{*}\left(\tilde{C}_{\theta}\right)=\varepsilon_{\tau(\theta)}$ for any $\theta \in \tilde{\varepsilon}$. Coordinates in the fiber of $\tau$ are called nonlocal variables.

In this paper we consider equations possessing two independent variables $x, t$ and two dependent variables $u, v$, i.e., $m=n=2$ in (3). The WE prolongation structures correspond to the cases when $\tau$ is a trivial bundle, i.e.,

$$
\tau: \varepsilon \times W \rightarrow \varepsilon,
$$

where $W$ is a finite dimensional manifold. Set $\operatorname{dim} W=l$, then the local coordinates $w^{1}, \cdots, w^{l}$ in $\mathrm{W}$ correspond to pseudopotentials in the WE prolongations approach [6] [7].

In coordinates, the above definition means that the total derivatives on $\tilde{\varepsilon} \subset J^{\infty}(2,2)$ are of the form

$$
\tilde{D}_{x}=D_{x}+X, \quad \tilde{D}_{t}=D_{t}+T,
$$

where $X$ and $T$ are $\tau$-vertical vector fields:

$$
X=\sum_{i=1}^{l} X_{i} \frac{\partial}{\partial w^{i}}, \quad T=\sum_{i=1}^{l} T_{i} \frac{\partial}{\partial w^{i}}
$$

Since the distribution on $\tilde{\varepsilon}$ is integrable, we have

$$
\left[\tilde{D}_{x}, \tilde{D}_{t}\right]=D_{x}(T)-D_{t}(X)+[X, T]=0
$$

If the coefficients $X^{i}, T^{j}$ in the vertical vector fields $X$ and $T$ are independent of nonlocal variables $w^{i}, i=1, \cdots, l$, then the condition (8) reduces to

$$
D_{x}(T)-D_{t}(X)=0 \text {, }
$$

the corresponding covering is called Abelian.

\section{The Prolongation Structures of the Reaction-Diffusion System}

We have introduced the covering theory for prolongation structure of nonlinear evolution equation in the previous section. Based upon this theory, we will discuss the corresponding prolongation structure for the system (1) in this section. The system have two independent variables $x, t$ and two dependent variables $u, v$, i.e., $m=n=2$ in (3). For convenience we rewrite the system as follows:

$$
\left\{\begin{array}{l}
u_{t}=u_{x x}-2 u^{2} v+2 k u \\
v_{t}=-v_{x x}+2 u v^{2}+2 k v
\end{array}\right.
$$

Then the corresponding jet space is $J^{\infty}(2,2)$ with the coordinates 


$$
x, t, u_{k}, v_{j}, \quad k \geq 0, \quad j \geq 0
$$

and the total derivative operators are given by

$$
\begin{gathered}
D_{x}=\frac{\partial}{\partial x}+\sum_{i \geq 0} u_{i+1} \frac{\partial}{\partial u_{i}}+\sum_{i \geq 0} v_{i+1} \frac{\partial}{\partial v_{i}}, \\
D_{t}=\frac{\partial}{\partial t}+\sum_{i \geq 0} D_{x}^{i}\left(u_{2}-2 u^{2} v+2 k u\right) \frac{\partial}{\partial u_{i}}+\sum_{i \geq 0} D_{x}^{i}\left(-v_{2}+2 u v^{2}-2 k v\right) \frac{\partial}{\partial v_{i}}, \\
\text { where } u_{k}=\frac{\partial^{k} u}{\partial x^{k}}, v_{j}=\frac{\partial^{j} v}{\partial x^{j}} .
\end{gathered}
$$

Set the covering for (1) is given by (6). By the integrability condition (8), we have

$$
\begin{aligned}
0= & {\left[\tilde{D}_{x}, \tilde{D}_{t}\right]=D_{x}(T)-D_{t}(X)+[X, T]=\frac{\partial T^{j}}{\partial x} \frac{\partial}{\partial w^{j}}+\sum_{i \geq 0} u_{i+1} \frac{\partial T^{j}}{\partial u_{i}} \frac{\partial}{\partial w^{j}} } \\
& +\sum v_{i+1} \frac{\partial T^{j}}{\partial v_{i}} \frac{\partial}{\partial w^{j}}-\frac{\partial X^{j}}{\partial t} \frac{\partial}{\partial w^{j}}-\sum_{i \geq 0} D_{x}^{i}\left(u_{2}-2 u^{2} v+2 k u\right) \frac{\partial X^{j}}{\partial u_{i}} \frac{\partial}{\partial w^{j}} \\
& -\sum_{i \geq 0} D_{x}^{i}\left(-v_{2}+2 u v^{2}-2 k v\right) \frac{\partial X^{j}}{\partial v_{i}} \frac{\partial}{\partial w^{j}}+[X, T]
\end{aligned}
$$

Now we consider the WE type coverings for (1) and suppose that both $X$ and $T$ are independent of $x, t$, i.e.,

$$
X=X\left(w^{1}, \cdots, w^{l}, u, v, u_{x}, v_{x}\right), \quad T=T\left(w^{1}, \cdots, w^{l}, u, v, u_{x}, v_{x}\right) .
$$

Then the equation (6) becomes

$$
\begin{aligned}
& 0=u_{1} \frac{\partial T^{i}}{\partial u} \frac{\partial}{\partial w^{i}}+u_{2} \frac{\partial T^{i}}{\partial u_{1}} \frac{\partial}{\partial w^{i}}+v_{1} \frac{\partial T^{i}}{\partial v} \frac{\partial}{\partial w^{i}}+v_{2} \frac{\partial T^{i}}{\partial v} \frac{\partial}{\partial w^{i}} \\
& -\left(u_{2}-2 u^{2} v+2 k u\right) \frac{\partial X^{i}}{\partial u} \frac{\partial}{\partial w^{i}}-\left(u_{3}-4 u u_{1} v-2 u^{2} v_{1}+2 k u_{1}\right) \frac{\partial X^{i}}{\partial u_{1}} \frac{\partial}{\partial w^{i}} \\
& -\left(-v_{2}+2 u v^{2}-2 k v\right) \frac{\partial X^{i}}{\partial v} \frac{\partial}{\partial w^{i}}-\left(-v_{3}+2 u_{1} v^{2}+4 u v v_{1}-2 k v_{1}\right) \frac{\partial X^{i}}{\partial v_{1}} \frac{\partial}{\partial w^{i}}+[X, T]
\end{aligned}
$$

Notice that the right hand side of the above equation is a polynomial in $u_{3}, v_{3}, u_{2}, v_{2}$. Therefore the coefficients at $u_{3}, v_{3}, u_{2}, v_{2}$ must vanish. Consequently we get

$$
\begin{gathered}
-\frac{\partial X^{i}}{\partial u_{1}}=0, \frac{\partial X^{i}}{\partial v_{1}}=0 \\
\frac{\partial T^{i}}{\partial u_{1}}-\frac{\partial X^{i}}{\partial u}=0, \frac{\partial T^{i}}{\partial v_{1}}+\frac{\partial X^{i}}{\partial v}=0 \\
u_{1} \frac{\partial T^{i}}{\partial u} \frac{\partial}{\partial w^{i}}+v_{1} \frac{\partial T^{i}}{\partial v} \frac{\partial}{\partial w^{i}}+\left(2 u^{2} v-2 k u\right) \frac{\partial X^{i}}{\partial u} \frac{\partial}{\partial w^{i}} \\
-\left(2 u v^{2}-2 k v\right) \frac{\partial X^{i}}{\partial v} \frac{\partial}{\partial w^{i}}+[X, T]=0
\end{gathered}
$$

By (16), $X$ is independent of $u_{1}, v_{1}$, hence $X=X\left(w^{1}, \cdots, w^{l}, u, v\right)$. By (17), $T$ can be expressed in the following form:

$$
T=u_{1} \frac{\partial X}{\partial u}-v_{1} \frac{\partial X}{\partial v}+R\left(w^{1}, \cdots, w^{l}, u, v\right)
$$


Substituting (19) into (18), we get

$$
\begin{aligned}
0= & u_{1}^{2} \frac{\partial^{2} X}{\partial u^{2}}+u_{1} \frac{\partial R}{\partial u}-v_{1}^{2} \frac{\partial^{2} X}{\partial v^{2}}+v_{1} \frac{\partial R}{\partial v}+\left(2 u^{2}-2 k u\right) \frac{\partial X}{\partial u} \\
& -\left(2 u v^{2}-2 k v\right) \frac{\partial X}{\partial v}+u_{1}\left[X, \frac{\partial X}{\partial u}\right]-v_{1}\left[X, \frac{\partial X}{\partial v}\right]+[X, R] .
\end{aligned}
$$

Similarly we can regard the left hand side of the above equation as a polynomial in $u_{1}, v_{1}$. Hence the coefficients at $u_{1}, v_{1}$ must vanish. Accordingly we get

$$
\begin{gathered}
\frac{\partial^{2} X}{\partial u^{2}}=0, \quad \frac{\partial^{2} X}{\partial v^{2}}=0, \\
\frac{\partial R}{\partial u}+\left[X, \frac{\partial X}{\partial u}\right]=0, \frac{\partial R}{\partial v}-\left[X, \frac{\partial X}{\partial u}\right]=0 \\
\left(2 u^{2} v-2 k u\right) \frac{\partial X}{\partial u}-\left(2 u v^{2}-2 k v\right) \frac{\partial X}{\partial v}+[X, R]=0
\end{gathered}
$$

Since $\frac{\partial^{2} X}{\partial u^{2}}=0, \frac{\partial^{2} X}{\partial v^{2}}=0$.

From (21) $X$ can be written as follows:

$$
X=A u v+B u+C v+D,
$$

where $A, B, C, D$ are only dependent on nonlocal variables $w_{1}, \cdots, w_{l}$. Substituting (24) into (22), we get:

$$
\begin{aligned}
& \frac{\partial R}{\partial u}=[A, C] v^{2}+[A, D] v+[B, C] v+[B, D] \\
& \frac{\partial R}{\partial v}=[B, A] u^{2}+[D, A] u+[B, C] u+[D, C]
\end{aligned}
$$

By (25) we have

$$
\begin{aligned}
& \frac{\partial^{2} R}{\partial u \partial v}=2[A, C] v+[A, D]+[B, C] \\
& \frac{\partial^{2} R}{\partial v \partial u}=2[B, A] u+[D, A]+[B, C] .
\end{aligned}
$$

Since $\frac{\partial^{2} R}{\partial u \partial v}=\frac{\partial R}{\partial v \partial u}$, we have

$$
[A, D]=0, \quad[A, C]=0, \quad[B, A]=0
$$

Putting the above condition into (25), we have

$$
\frac{\partial R}{\partial u}=[B, C] v+[B, D], \quad \frac{\partial R}{\partial v}=[B, C] u+[D, C]
$$

Hence

$$
R=[B, C] u v+[B, D] u+[D, C] v+E,
$$

where $E$ is only dependent on nonlocal variables, i.e., $E=E\left(w_{1}, \cdots, w_{l}\right)$.

Substituting (29) and (24) into (19), we have

$$
T=A u_{1} v-A v_{1} u+B u_{1}-C v_{1}+[B, C] u v+[B, D] u+[D, C] v+E
$$

Substituting (30) and (24) into (23), we have 


$$
\begin{aligned}
& 2 B u^{2} v-2 C u v^{2}-2 k B u+2 k C v+[A, E] u v+[B,[B, C]] u^{2} v \\
& +[B,[B, D]] u^{2}+[B,[D, C]] u v+[B, E] u+[C,[B, C]] u v^{2} \\
& +[C,[B, D]] u v+[C,[D, C]] v^{2}+[C, E] v+[D,[B, C]] u v \\
& +[D,[B, D]] u+[D,[D, C]] v+[D, E]=0
\end{aligned}
$$

The right hand side of the above equality is a polynomial in $u, v$. Thus we have

$$
\begin{gathered}
2 B+[B,[B, C]]=0,[B,[B, D]]=0,[C,[D, C]]=0,[C,[B, C]]-2 C=0 \\
{[B, E]-2 k B+[D,[B, D]]=0,2 k C+[C, E]+[D,[D, C]]=0,[D, E]=0} \\
{[A, E]+[B,[D, C]]+[C,[B, D]]+[D,[B, C]]=[A, E]+2[D,[B, C]]=0 .}
\end{gathered}
$$

Hence we have proved the following statement:

Theorem 3.1. For the reaction-diffusion system (1), any WE prolongation type coverings

$$
\begin{gathered}
D_{x}(T)-D_{t}(X)+[X, T]=0, \\
X=X\left(w^{1}, \cdots, w^{l}, u, v, u_{x}, v_{x}\right), \quad T=T\left(w^{1}, \cdots, w^{l}, u, v, u_{x}, v_{x}\right)
\end{gathered}
$$

are given by

$$
\begin{gathered}
X=A u v+B u+C v+D, \\
T=A u_{1} v-A v_{1} u+B u_{1}-C v_{1}+[B, C] u v+[B, D] u+[D, C] v+E
\end{gathered}
$$

where the vector fields $A, B, C, C, E$ are all depend on nonlocal variables $w^{1}, \cdots, w^{l}$ only and satisfy the following brackets

$$
\begin{gathered}
2 B+[B,[B, C]]=0,[B,[B, D]]=0,[C,[D, C]]=0,[C,[B, C]]-2 C=0 \\
{[B, E]-2 k B+[D,[B, D]]=0,2 k C+[C, E]+[D,[D, C]]=0,[D, E]=0} \\
{[A, E]+2[D,[B, C]]=0,[A, D]=0,[A, C]=0,[B, A]=0 .}
\end{gathered}
$$

Next we will discuss about the realizations and classifications of one dimensional WE coverings of (1).

Assume $A \neq 0$, then a suitable nonlocal variable $w$ can be chosen such that $A=\frac{\partial}{\partial w}$. Let $B=\beta \frac{\partial}{\partial w}, C=\gamma \frac{\partial}{\partial w}, D=\delta \frac{\partial}{\partial w}, E=\epsilon \frac{\partial}{\partial w}$, where $\beta, \gamma, \delta, \epsilon$ are dependent on $w$ only. By Theorem (3.1), we can get

$$
B=0, C=0, D=\delta \frac{\partial}{\partial w}, E=\epsilon \frac{\partial}{\partial w}
$$

where $\delta, \epsilon$ are constants. Meanwhile, when this happens, all the brackets in Theorem (3.1) are satisfied automatically.

Hence the above covering is equivalent to

$$
X=(u v+\delta) \frac{\partial}{\partial w}, \quad T=\left(u_{1} v-u v_{1}+\epsilon\right) \frac{\partial}{\partial w},
$$

where $\delta, \epsilon$ are constants.

Secondly let $A=0$. Then we will consider $B \neq 0$ and $B=0$ respectively.

1. Assume that $B \neq 0$ then we can choose a suitable variable $w$ in such a way that $B=\frac{\partial}{\partial w}$. By (36), we have 


$$
C=\left(-w^{2}+\alpha w+\beta\right) \frac{\partial}{\partial w}, \quad D=(p w+q) \frac{\partial}{\partial w},
$$

where $\alpha, \beta, p, q$ satisfying that

$$
\alpha^{2}+4 \beta=0,2 q+\alpha p=0 .
$$

Substituting (40) into (37) and (38), we have

$$
E=r(p w+q) \frac{\partial}{\partial w},
$$

where $r>0$ satisfying that

$$
\left\{\begin{array}{l}
p^{2}-p r+2 k=0 \\
k \alpha+q(r-p)=0 \\
2 k \beta+\beta r p-\alpha q r-2 q^{2}-p q \alpha+p^{2} \beta=0
\end{array}\right.
$$

Notice that the latter two constraints of the above formula can be deduced from the first one and (41). Hence, when $A=0, B=\frac{\partial}{\partial w}$, we have:

$$
C=\left(-w^{2}+\alpha w+\beta\right) \frac{\partial}{\partial w}, D=(p w+q) \frac{\partial}{\partial w}, E=r(p w+q) \frac{\partial}{\partial w}
$$

where $\alpha, \beta, p, q, r$ satisfying

$$
\left\{\begin{array}{l}
\alpha^{2}=-4 \beta \\
2 q+\alpha p=0 \\
p^{2}-p \gamma+2 k=0
\end{array}\right.
$$

Furthermore, the above covering is equivalent to

$$
\begin{gathered}
X=\left(u-v w^{2}+2 w v+\beta v+p w+q\right) \frac{\partial}{\partial w} \\
T=\left[u_{1}-v_{1}\left(-w^{2}+\alpha w+\beta\right)+(-2 w+\alpha) u v\right. \\
\left.+p u+\left(-p w^{2}-2 q w+p \beta\right) v+r(p w+q)\right] \frac{\partial}{\partial w},
\end{gathered}
$$

where $\alpha, \beta, p, q, r$ satisfying (45).

2. If $A=0, B=0$, then from Theorem (3.1), we know that $C=0$. Hence the covering is trivial.

In summary, we have proved the following result from the above discussion:

Theorem 3.2. For the reaction-diffusion system (1), any WE prolongation type coverings are locally equivalent to one of the followings:

$$
\begin{aligned}
\tau^{1} & : \tilde{D}_{x}=\frac{\partial}{\partial x}+\sum_{i \geq 0} u_{i+1} \frac{\partial}{\partial u_{i}}+\sum_{i \geq 0} v_{i+1} \frac{\partial}{\partial v_{i}}+(u v+\delta) \frac{\partial}{\partial w}, \\
\tilde{D}_{t}= & \frac{\partial}{\partial t}+\sum_{i \geq 0} D_{x}^{i}\left(u_{2}-2 u^{2} v+2 k u\right) \frac{\partial}{\partial u_{i}} \\
& +\sum_{i \geq 0} D_{x}^{i}\left(-v_{2}+2 u v^{2}-2 k v\right) \frac{\partial}{\partial v_{i}}+\left(u_{1} v-u v_{1}+\epsilon\right) \frac{\partial}{\partial w},
\end{aligned}
$$

where $\delta, \epsilon$ are constants.

$$
\tau^{2}: \tilde{D}_{x}=\frac{\partial}{\partial x}+\sum_{i \geq 0} u_{i+1} \frac{\partial}{\partial u_{i}}+\sum_{i \geq 0} v_{i+1} \frac{\partial}{\partial v_{i}}+\left(u-v w^{2}+2 w v+\beta v+p w+q\right) \frac{\partial}{\partial w},
$$




$$
\begin{aligned}
\tilde{D}_{t}= & \frac{\partial}{\partial t}+\sum_{i \geq 0} D_{x}^{i}\left(u_{2}-2 u^{2} v+2 k u\right) \frac{\partial}{\partial u_{i}}+\sum_{i \geq 0} D_{x}^{i}\left(-v_{2}+2 u v^{2}-2 k v\right) \frac{\partial}{\partial v_{i}} \\
+ & {\left[u_{1}-v_{1}\left(-w^{2}+\alpha w+\beta\right)+(-2 w+\alpha) u v+p u\right.} \\
& \left.+\left(-p w^{2}-2 q w+p \beta\right) v+r(p w+q)\right] \frac{\partial}{\partial w},
\end{aligned}
$$

where $\alpha, \beta, p, q, r$ satisfying (45).

Remark 3.1. Obviously $\tau^{1}$ is not dependent on nonlocal variable $w$, hence it is Abelian covering for the system (1). By Theorem (2.1), we can obtain a conservation law for the reaction-diffusion system (1):

$$
\omega=(u v+\delta) \mathrm{d} x+\left(u_{1} v-u v_{1}+\epsilon\right) \mathrm{d} t
$$

\section{Concluding Remarks}

We have investigated prolongation structure for the system (1) by using the covering theory. For this prolongation structure theory, the realizations and the classifications of the one-dimensional coverings of the system can be obtained. By comparison with the result by Alfinito et al. [1], we find they are actually the same. Here we are from the point view of tangent bundle theory, but WE prolongation theory is based on the cotangent bundle theory. Other than that, we can easily get the corresponding conservation law from the one-dimensional Abelian coverings.

It should be mentioned that a lot of questions remain to be understood. Firstly, it should be important to try to extend the prolongation technique to the study of higher dimension nonlinear field equations. For example, the more general (2 +1 )-dimensional reaction $C$ diffusion equations can be written as

$$
\left\{\begin{array}{l}
u_{t}=D_{1} \Delta u+b_{1} u^{2} v+b_{2} u v^{2}+b_{3} u+b_{4} v+b_{5} \\
v_{t}=D_{2} \Delta v+c_{1} u^{2} v+c_{2} u v^{2}+c_{3} u+c_{4} v+c_{5}
\end{array}\right.
$$

where $u=u(x, y, t), v=v(x, y, t), \Delta$ is the Laplace operator in two-dimensional orthonormal coordinates; $D_{1}$ and $D_{2}$ are the diffusion constants; $b_{i}$ and $c_{i}, i=1,2, \cdots, 5$, are the coefficients. How to give the corresponding prolongation structure? Secondly, another important aspect which deserves to be explored is the comparison between the covering theory and covariant theory [2]. Thirdly, how to obtain the realizations and the classifications of higher dimensional coverings of the system? Some of them will be in the forthcoming publication.

\section{Acknowledgements}

The author is partially supported by the natural science foundation of Fujian Province (2013J01027) and is very thankful for everything.

\section{References}

[1] Alfinito, E., Grassi, V., Leo, R.A., et al. (1998) Equations of the Reaction-Diffusion Type with a Loop Algebra Structure. Inverse Problems, 14, 1387-1401. https://doi.org/10.1088/0266-5611/14/6/003

[2] Duan, X.J., Deng, M., Zhao, W.Z., et al. (2007) The Prolongation Structure of the 
Inhomogeneous Equation of the Reaction Diffusion Type. Journal of Physics $A$ Mathematical and Theoretical, 40, 3831-3837. https://doi.org/10.1088/1751-8113/40/14/006

[3] Martina, L., Pashaev, O.K. and Soliani, G. (1997) Integrable Dissipative Structures in the Gauge Theory of Gravity. Classical and Quantum Gravity, 14, 3179. https://doi.org/10.1088/0264-9381/14/12/005

[4] Ding, Q., Inoguchi, J.I. (2004) Schrodinger Flows, Binormal Motion for Curves and the Second AKNS-Hierarchies. Chaos Solitons and Fractals, 21, 669-677. https://doi.org/10.1016/j.chaos.2003.12.092

[5] Zhao, W.-Z., Li, M.-L., Qi, Y.-H. and Wu, K. (2005) Modified Heisenberg Ferromagnet Model and Integrable Equation. Communications in Theoretical Physics, 44, 415-419. https://doi.org/10.1088/6102/44/3/415

[6] Wahlquist, H.D. and Estabrook, F.B. (1975) Prolongation Structures of Nonlinear Evolution Equations. Journal of Mathematical Physics, 16, 1-7. https://doi.org/10.1063/1.522396

[7] Wahlquist, H.D. and Estabrook, F.B. (1976) Prolongation Structures of Nonlinear Evolution Equations II. Journal of Mathematical Physics, 17, 1293-1297. https://doi.org/10.1063/1.523056

[8] Krasil'shchik, J. and Verbovetsky, A. (2012) Geometry of Jet Spaces and Integrable Systems. Journal of Geometry and Physics, 61, 1633-1674.

https://doi.org/10.1016/j.geomphys.2010.10.012

[9] Krasil'Shchik, I.S. and Vinogradov, A.M. (1989) Nonlocal Trends in the Geometry of Differential Equations: Symmetries, Conservation Laws, and Backlund Transformations. Acta Applicandae Mathematicae, 15, 161-209. https://doi.org/10.1007/BF00131935

[10] Krasil'shchik, I.S., Lychagin, V.V. and Vinogradov, A.M. (1986) Geometry of jet Spaces and Nonlinear Partial Differential Equations. Gordon and Breach Science Publishers, Philadelphia.

[11] Vinogradov, A.M. (1984) Local Symmetries and Conservation Laws. Acta Applicandae Mathematicae, 2, 21-78. https://doi.org/10.1007/BF01405491

[12] Krasilshchik, I.S. and Vinogradov, A.M. (1984) Nonlocal Symmetries and the Theory of Coverings: An Addendum to A. M. Vinogradov's 'Local Symmetries and Conservation Laws? Acta Applicandae Mathematicae, 2, 79-96.

https://doi.org/10.1007/BF01405492

[13] Krasil'Shchik, I.S. and Vinogradov, A.M. (1989) Nonlocal Trends in the Geometry of Differential Equations: Symmetries, Conservation Laws, and Backlund Transformations. Acta Applicandae Mathematicae, 15, 161-209. https://doi.org/10.1007/BF00131935

[14] Krasil'Shchik, I.S., Verbovetsky, A.M. and Vitolo, R. (2012) A Unified Approach to Computation of Integrable Structures. Acta Applicandae Mathematicae, 120, 199218. https://doi.org/10.1007/s10440-012-9699-x

[15] Cheng, Q. and Jingsong, H.E. (2014) The Prolongation Structures and Nonlocal Symmetries for Modified Boussinesq System. Acta Mathematica Scientia, 34B, 215 227. https://doi.org/10.1016/S0252-9602(13)60139-3 
Submit or recommend next manuscript to SCIRP and we will provide best service for you:

Accepting pre-submission inquiries through Email, Facebook, LinkedIn, Twitter, etc. A wide selection of journals (inclusive of 9 subjects, more than 200 journals)

Providing 24-hour high-quality service

User-friendly online submission system

Fair and swift peer-review system

Efficient typesetting and proofreading procedure

Display of the result of downloads and visits, as well as the number of cited articles Maximum dissemination of your research work

Submit your manuscript at: http://papersubmission.scirp.org/

Or contact jamp@scirp.org 